\title{
openheart Distractions in the cardiac catheterisation laboratory: impact for cardiologists and patient safety
}

\author{
Kalaivani Mahadevan (D) , ${ }^{1}$ Elena Cowan (D) , ${ }^{1}$ Navneet Kalsi, ${ }^{2}$ Helena Bolam, ${ }^{1}$ \\ Richard Arnett, ${ }^{3}$ Alex Hobson, ${ }^{1}$ Kaushik Guha, ${ }^{1}$ Geraint Morton, ${ }^{1}$
} Peter A Brennan, ${ }^{4,5}$ Paul R Kalra ${ }^{1,5}$

\begin{abstract}
- Additional material is published online only. To view, please visit the journal online (http://dx.doi.org/10.1136/ openhrt-2020-001260).
\end{abstract}

To cite: Mahadevan K, Cowan E, Kalsi N, et al. Distractions in the cardiac catheterisation laboratory: impact for cardiologists and patient safety. Open Heart 2020;7: 001260. doi:10.1136/

openhrt-2020-001260

Received 3 February 2020 Revised 3 September 2020 Accepted 3 September 2020

Check for updates

(C) Author(s) (or their employer(s)) 2020. Re-use permitted under CC BY-NC. No commercial re-use. See rights and permissions. Published by BMJ.

${ }^{1}$ Cardiology Department, Queen Alexandra Hospital, Portsmouth Hospitals University NHS Trust, Portsmouth, UK

${ }^{2}$ Royal Hampshire County Hospital, Winchester, UK ${ }^{3}$ Quality Enhancement Office, Royal College of Surgeons in Ireland, Dublin, Ireland ${ }^{4}$ Maxillofacial Surgery Department, Queen Alexandra Hospital, Portsmouth Hospitals University NHS Trust,

Portsmouth, UK

${ }^{5}$ University of Portsmouth,

Portsmouth, UK

Correspondence to

Dr Elena Cowan; cowan.elena@ gmail.com

\section{ABSTRACT}

Objective To understand human factors (HF) contributing to disturbances during invasive cardiac procedures, including frequency and nature of distractions, and assessment of operator workload.

Methods Single centre prospective observational evaluation of 194 cardiac procedures in three adult cardiac catheterisation laboratories over 6 weeks. A proforma including frequency, nature, magnitude and level of procedural risk at the time of each distraction/ interruption was completed for each case. The primary operator completed a National Aeronautical and Space Administration (NASA) task load questionnaire rating mental/physical effort, level of frustration, time-urgency, and overall effort and performance.

Results 264 distractions occurred in 106 (55\%) out of 194 procedures observed; $80 \%$ were not relevant to the case being undertaken; $14 \%$ were urgent including discussions of potential ST-elevation myocardial infarction requiring emergency angioplasty. In procedures where distractions were observed, frequency per case ranged from 1 to 16 (mean 2.5, SD \pm 2.2 ); 43 were documented during high-risk stages of the procedure. Operator rating of NASA task load parameters demonstrated higher levels of mental and physical workload and effort during cases in which distractions occurred.

Conclusions In this first description of HF in adult cardiac catheter laboratories, we found that fewer than half of all procedures were completed without interruption/ distraction. The majority were unnecessary and without relation to the case or list. We propose the introduction of a 'sterile cockpit' environment within catheter laboratories, as adapted from aviation and used in surgical operating theatres, to minimise non-emergent interruptions and disturbances, to improve operator conditions and overall patient safety.

\section{INTRODUCTION}

There is growing recognition of the importance of human factors (HF) in healthcare and patient safety. ${ }^{12}$ Better understanding of working environments is paramount to improving clinical working conditions and patient safety. Distractions and interruptions are common in clinical medicine. Their

\section{Key questions}

What is already known about this subject?

- Distractions and interruptions are increasingly recognised in clinical medicine and may have adverse impact on staff performance and patient safety.

What does this study add?

- First description of significant levels of non-urgent and case irrelevant distractions in adult cardiac catheterisation laboratory and their association with increased perception of workload in cardiologists.

How might this impact on clinical practice?

- Non-urgent distractions in cardiac catheterisation laboratory should be minimised. A 'sterile cockpit' environment with only essential case-relevant communication and no interruptions during critical stages of procedures may be considered.

negative impact on staff performance has been recognised across a number of clinical areas. ${ }^{3-8}$ There is an inverse relationship between interruptions and miscommunications during surgical procedures. ${ }^{5}$ Interruptions and multitasking are associated with significantly increased prescribing errors in emergency departments. ${ }^{6}$ A systematic review showed auditory and mental distractions in laboratory/simulation studies can adversely impact surgical performance. ${ }^{7}$ In addition, intraoperative distractions led to fewer safety checks by urological surgical teams. ${ }^{8}$ In contrast, the use of a 'do not interrupt' intervention bundle led to a significant decrease in non-medication related interruptions to nursing staff during medication administration. ${ }^{9}$

Surgical specialties have led the translation of HF research, alongside safety concepts from the aviation industry, into clinical practice. ${ }^{1}$ For example, the WHO 'Surgical Safety Checklist' has been widely adopted 
in operating theatres for over a decade but only more recently in cardiac catheterisation laboratories. ${ }^{10}$

The cardiac catheter laboratory shares similarities with the operating room. A primary operator and 4-6 other healthcare professionals work as a team to investigate/ treat patients, in often emergent situations, with complex invasive procedures. Asides from incorporation of the generic WHO checklist, there has been no specific evaluation of the impact of HF in adult catheter laboratories. We set out to describe the frequency and nature of distractions in the adult cardiac catheter laboratory and their impact on cardiologists and patients.

\section{METHODS}

We undertook a 6-week single centre prospective observational patient safety and service evaluation project (July to September 2018) in our three adult cardiac catheterisation laboratories. Activity included primary percutaneous coronary intervention (PPCI), percutaneous coronary intervention (PCI), coronary angiography (CA), device implantation-permanent pacemaker (PPM), implantable cardioverter defibrillator (ICD) and cardiac resynchronisation therapy (CRT), electrophysiological studies (EPS) and ablation.

The casemix included emergencies (ST-elevation myocardial infarction (STEMI), pericardial tamponade, unstable heart block), acute in-patient and elective outpatient procedures. Catheter laboratory team members were informed prior to commencement of the evaluation period including discussion around data collection with reminders at daily team brief. Physiologists, noted to have the best overall 'birds-eye' perspective of the catheter laboratory, were requested to complete a proforma for each case, including duration of procedure, frequency and nature of distractions and team members involved.

When possible, the risk stage of the procedure at time of distraction was also noted (table 1).

\section{Distraction coding and National Aeronautical and Space Administration task load index}

Distracting sources were audited prior to derivation of a formal distraction source list (table 2).

Background music was classified as a distraction if any team member requested volume reduction/cessation. The magnitude of distraction was coded as per the Healey Scoring System from least to most disruptive (table 3, observed effect). ${ }^{11}$

Risk stages (table 1), modelled in similar fashion to that proposed by Bergersen et al in their study of paediatric and congenital cardiac catheterisation, ${ }^{12}$ were classified into four groups, ranging from category 1 (vascular access and wound closure) to category 4 (cardiac arrest, tamponade). The anticipation being that an increase in risk category would demand an increase in catheter laboratory team concentration.

Procedural duration was classified as category 1 ( $\leq 30 \mathrm{~min})$, category 2 (31-60 $\mathrm{min})$; category 3 (61-120 min), category 4 (121-180 min), category 5 (181-240 min), category 6 (>240 min).

The Hart and Stavelands National Aeronautical and Space Administration Task load Index (NASA TLX, online supplemental figure 1), a widely used and robustly validated human-centred tool, was employed to assess operator workload. The primary operator was asked to

Table 1 Categorisation of risk stages of procedures performed in cardiac catheterisation laboratory

\begin{tabular}{|c|c|c|c|c|}
\hline & Risk category 1 & Risk category 2 & Risk category 3 & Risk category 4 \\
\hline Primary PCI & Vascular access & $\begin{array}{l}\text { Catheter exchange } \\
\text { Contrast delivery }\end{array}$ & $\begin{array}{l}\text { Critical time to open artery } \\
\text { Balloon inflation and stent delivery }\end{array}$ & $\begin{array}{l}\text { Cardiac arrest } \\
\text { Pericardial tamponade } \\
\text { Life-threatening bleed }\end{array}$ \\
\hline Elective angioplasty & Vascular access & Catheter exchange & Balloon inflation and stent delivery & $\begin{array}{l}\text { Cardiac arrest } \\
\text { Pericardial tamponade } \\
\text { Life-threatening bleed }\end{array}$ \\
\hline CRT & $\begin{array}{l}\text { Vascular access } \\
\text { Wound closure }\end{array}$ & $\begin{array}{l}\text { RV/RA lead placement } \\
\text { Access to coronary sinus }\end{array}$ & $\begin{array}{l}\text { Placement of LV lead/removal of } \\
\text { delivery sheath }\end{array}$ & $\begin{array}{l}\text { Cardiac arrest } \\
\text { Pericardial tamponade } \\
\text { Life-threatening bleed }\end{array}$ \\
\hline EPS/Ablation & Vascular access & $\begin{array}{l}\text { Catheter placement } \\
\text { Catheter exchange }\end{array}$ & $\begin{array}{l}\text { Ablation catheter } \\
\text { Signal analysis } \\
\text { Transeptal puncture }\end{array}$ & $\begin{array}{l}\text { Cardiac arrest } \\
\text { Pericardial tamponade } \\
\text { Life-threatening bleed }\end{array}$ \\
\hline Diagnostic angiogram & Vascular access & $\begin{array}{l}\text { Catheter exchange } \\
\text { Contrast delivery }\end{array}$ & & $\begin{array}{l}\text { Cardiac arrest } \\
\text { Pericardial tamponade } \\
\text { Life-threatening bleed }\end{array}$ \\
\hline Bradycardic PPM or ICD & $\begin{array}{l}\text { Vascular access } \\
\text { Wound closure }\end{array}$ & RV/RA lead placement & & $\begin{array}{l}\text { Cardiac arrest } \\
\text { Pericardial tamponade } \\
\text { Life-threatening bleed }\end{array}$ \\
\hline
\end{tabular}

CRT, cardiac resynchronisation therapy device implantation; EPS, electrophysiological studies; ICD, implantable cardioverter defibrillator implantation; LV, left ventricular; PCl, percutaneous coronary intervention; PPM, permanent pacemaker implantation; RA, right atrial; RV, right ventricular. 


\begin{tabular}{ll}
\hline Table 2 & Common distracting sources \\
\hline No & Potential distracting source \\
\hline 1 & Phone - in lab or in close proximity \\
\hline 2 & Bleeper \\
3 & $\begin{array}{l}\text { Radio - action or response to the radio causing } \\
\text { distraction }\end{array}$ \\
\hline 4 & Case irrelevant communication \\
5 & Communication difficulties \\
6 & External staff \\
7 & Equipment-provision or failure \\
8 & Working environment \\
9 & Procedural events \\
\hline
\end{tabular}

score task load components (effort, performance, mental demand, physical demand, frustration and temporal demand) on a 21-point graded scale from low to high, immediately post procedure to ensure perceptions were an accurate reflection of experience.

Patients completed an experience and satisfaction questionnaire (excluding those who received general anaesthesia) rating perceived quality and success of procedure (scale 1 (poor) to 10 (excellent)) and continuity of team members.

\section{Statistical analyses}

Procedural distraction data were analysed using the Rasch Rating Scale model which takes ordinal data and computes a linear (equal-interval) measure which can be used in further analyses. Rasch model analysis focuses on data quality in relation to the hypothesised construct (task load in this case) and produces a number of metrics which can be used to judge the validity of the measurement instrument and its resulting data. Data judged to fit the Rasch model can be regarded as interval data and analysed appropriately. The structure and functioning of the original 21 point response scale was evaluated and in order to maximise model fit, rating scale categories were merged to create a new 6-point scale.

Table 3 Grading of the interference or disturbance on the intended task/procedure

\begin{tabular}{ll}
\hline Event level & Observed effect \\
\hline 1 & Potentially distracting source \\
\hline 2 & Interference noticed by floating personnel \\
\hline 4 & Floating member attends to non-case interference \\
\hline 5 & Team member momentarily distracted from task \\
\hline 6 & Team member pauses current task \\
\hline 7 & Team member attends to distraction \\
\hline 8 & Whole/part team momentarily distracted from task \\
\hline 9 & Whole/part team attend to distraction \\
\hline
\end{tabular}

$1=$ least, $9=$ most.
After validation, the resulting data (measured in logits) represented a valid measurement of the construct of task load. Extreme values (maximum and minimum) were excluded as they did not provide any useful measurement information. The remaining data were normally distributed interval data and analysed using parametric methods.

To ensure an appropriate amount of data for analysis of impact of interruptions on the taskload, the structure of some descriptive variables were modified. Only procedure types where data were collected on $\geq 10$ cases were included (CA, CRT, PCI and PPCI). In terms of the number of distractions, all occurrences with $\geq 3$ distractions were included as a single category. For the duration of the procedure, all observations classified as category four or above were classified as $4+$.

The relationship between descriptive variables and task load was analysed using analysis of variance (ANOVA) with post hoc comparisons investigated using Tukey's 'Honest Significant Difference' (HSD) method which allows multiple pairwise comparisons between variable levels while correcting for the family-wise error rate .

\section{Patient and public involvement}

Patients/public were not involved in design, conduct, reporting or dissemination plans.

\section{RESULTS \\ Procedural distractions}

Data were collected from 194 procedures: EPS/ablation $(n=17)$, box change $(n=8)$, ICD/CRT implants $(n=13)$, coronary interventions ( $\mathrm{n}=148$, including 10 PPCI), PPM implant $(\mathrm{n}=8)$. The primary operator was a fellow (ST7) in $41 \%$, consultant in $58 \%$ and registrar in $1 \%$ of cases.

A total of 264 distractions occurred in $55 \%(n=106)$ of all procedures undertaken. In procedures where interruptions were observed, frequency per case ranged from $1(n=43)$ to $16(n=1)$.

An average of $2.5(\mathrm{SD} \pm 2.2)$ distractions were seen in cases when any distraction happened. The number of distractions occurring across procedure types is shown in figure 1. Eight out of 10 PPCI procedures were interrupted (1-8 interruptions per case) and 14 of the interruptions happened during stages of procedural difficulty 2-4 (i.e. after gaining vascular access).

Procedural duration was recorded in 189 procedures $(97 \%)$; 67 were duration category 1,63 - category 2 ; 33 category 3, 20-category 4, 4-category 5, 2-category 6 . Fifty eight per cent of distractions occurred in cases with procedural time $<120 \mathrm{~min}$.

Eighty per cent $(n=210)$ of all distractions had no relevance to the case underway at the time. Only $14 \%$ were urgent including discussions around potential STEMI/ PPCI. Online supplemental figure 2 shows distribution of distraction by source, with interruption of the catheter laboratory team by external staff accounting for $60 \%$. Free-text descriptions of individual distractions were 


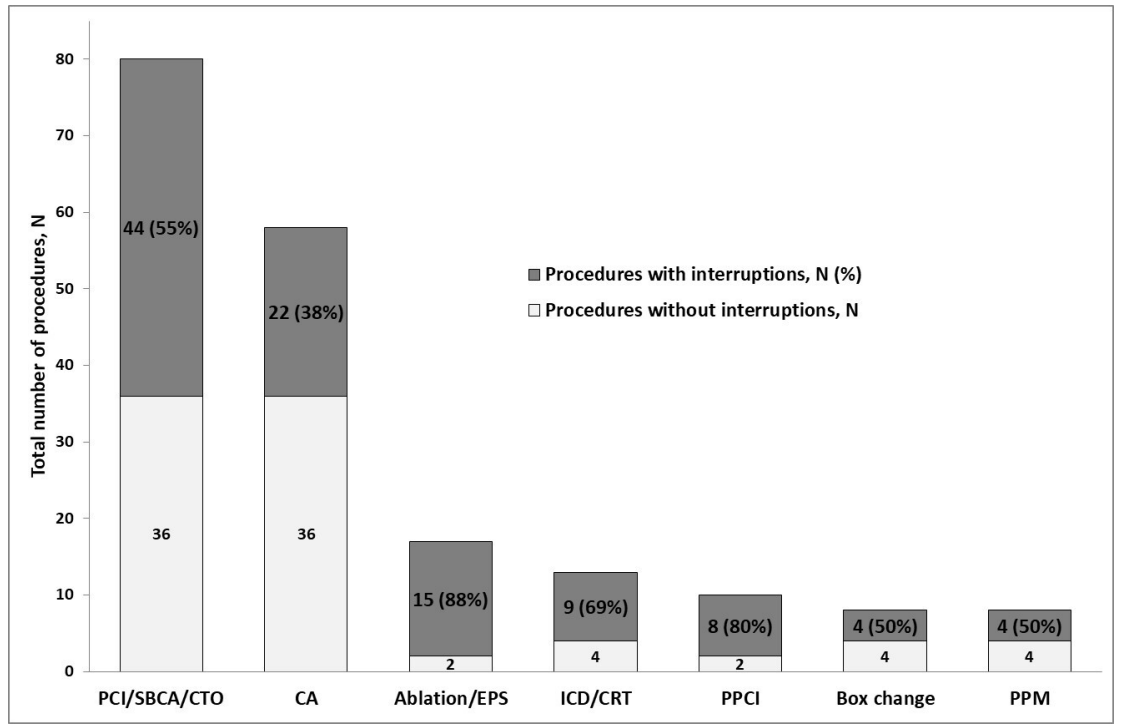

Figure 1 Distractions observed during different procedures. Number $(\mathrm{N})$ of procedures without interruptions and number and percentage $(\mathrm{N}, \%)$ of procedures where interruptions were observed. $\mathrm{CA}$, coronary angiography; $\mathrm{CTO}$, chronic total occlusion intervention; EPS, electrophysiological studies; ICD/CRT, implantable cardioverter defibrillator/cardiac resynchronisation therapy device implantation; $\mathrm{PCl}$, percutaneous coronary intervention; $\mathrm{PPCl}$, primary percutaneous coronary intervention; PPM, permanent pacemaker implantation; SBCA, standby coronary angiogram.

encouraged and included: 'discussion regarding practitioner finishing time,' 'coordinator entered to check progress of list,' 'ED call to discuss STEMI,' 'equipment request,' 'discussion of daily jobs,' 'discussion of stock,' 'registrar answering multiple bleeps,' 'radiographers/ physiologists discussing breaks.'

In $37 \%(n=97)$, the risk stage of the procedure at the time of distraction was recorded (online supplemental figure 3). Forty four per cent $(n=43)$ occurred during high-risk stages including critical time to balloon inflation and reperfusion in STEMI. On analysis of magnitude of distraction on the primary operator, we demonstrated transient but complete procedural cessation in $16 \%$ $(n=43)$ of all interruptions, of which nine were during emergencies.

\section{Validation of National Aeronautical and Space Administration task load index}

An initial evaluation of the 21-point rating scale suggested that it was not used uniformly or consistently by respondents and so individual rating scale points were collapsed into six categories that demonstrated a similar number of observations with distinct Rasch probability. This meant that the possible score for each item ranged from 1 to 6 and the entire instrument score ranged from 6 to 36 . Rasch analysis identified seven responses that did not fit the model and these were removed during calibration. After calibration, raw scores increased monotonically with overall task load measures and all six items demonstrated strong positive correlation with overall task load measures (range 0.61-0.87) suggesting a single construct (task load). Items demonstrated an appropriate level of model fit with both infit and outfit ranges between 0.5 and 1.5. Calibrated items had a logit difficulty range of between -1.3 and 1.3. On the task load scale, low logit values indicate items that are easier (more commonly attract higher ratings) and high logit values indicate items that are harder (less commonly attract higher ratings). The person reliability of the calibrated model was 0.87 (equivalent to a Cronbach's Alpha value of 0.89) with a separation index of 2.59. The item reliability was 0.99 suggesting that the responses were sufficient to confidently establish the item hierarchy.

\section{Relationship between task load and case variables}

The resulting task load measurements were analysed in relation to other case related variables. The results of a t-test/one-way ANOVAs suggested a difference in mean task load scores related to the presence of distractions $(\mathrm{t}=-4.26, \mathrm{df}=171.1, \mathrm{p}<0.01)$; number of distractions $\left(\mathrm{F}_{(3,173)}=7.79, \mathrm{p}<0.001\right)$; type of procedure $\left(\mathrm{F}_{(3,134)}=11.28\right.$, $\mathrm{p}<0.001)$ and duration of procedure $\left(\mathrm{F}_{(3,169)}=25.30\right.$, $\mathrm{p}<0.001)$. The results of subsequent post hoc Tukey HSD tests are shown in figure 2.

There was evidence of higher task load scores in cases with one or more distractions as compared with no distractions and in cases with three or more distractions as compared with no distractions. Higher mean task load scores were seen for PPCI, PCI and CRT compared with CA. A trend towards higher mean task load scores was seen with increasing procedural duration (with the exception of category 2 vs 3). Predictably ICD/CRT implantation was associated with longer procedural duration with 12 out of 13 lasting $>60 \mathrm{~min}$ and 1-8 interruptions per case in approximately $70 \%(\mathrm{n}=9)$.

\section{Patient experience}

Patient rating of procedural experience and success was recorded in $93 \%$ of cases. Mean satisfaction score in the 

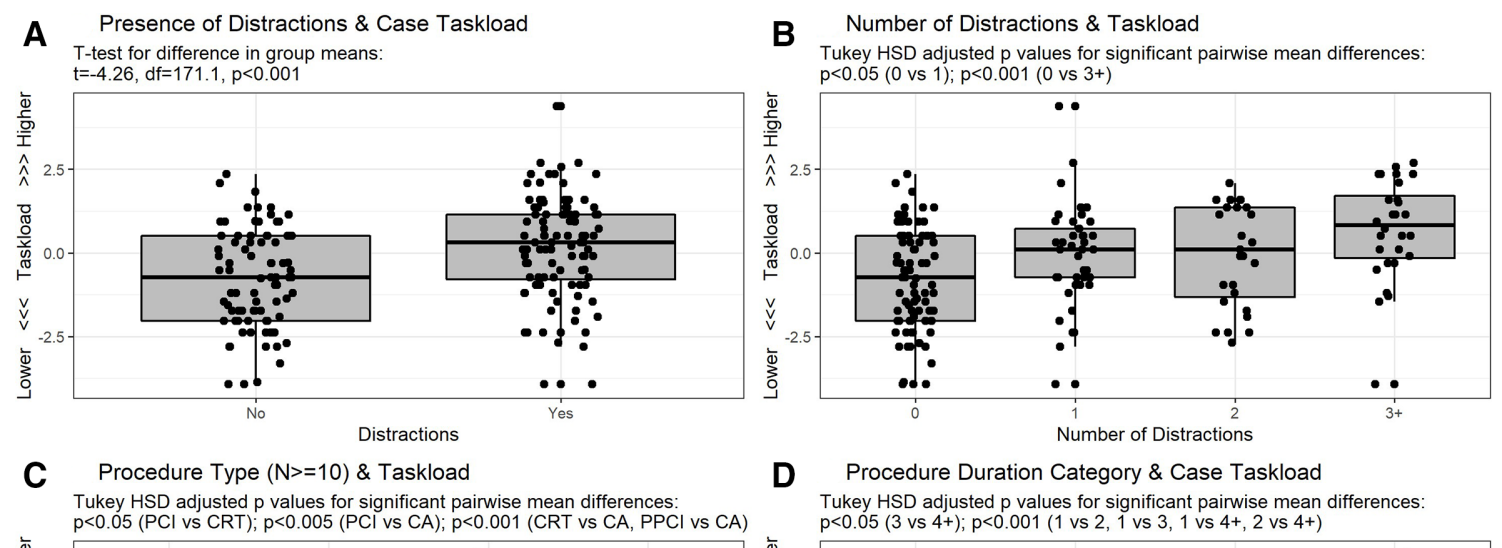

D Procedure Duration Category \& Case Taskload
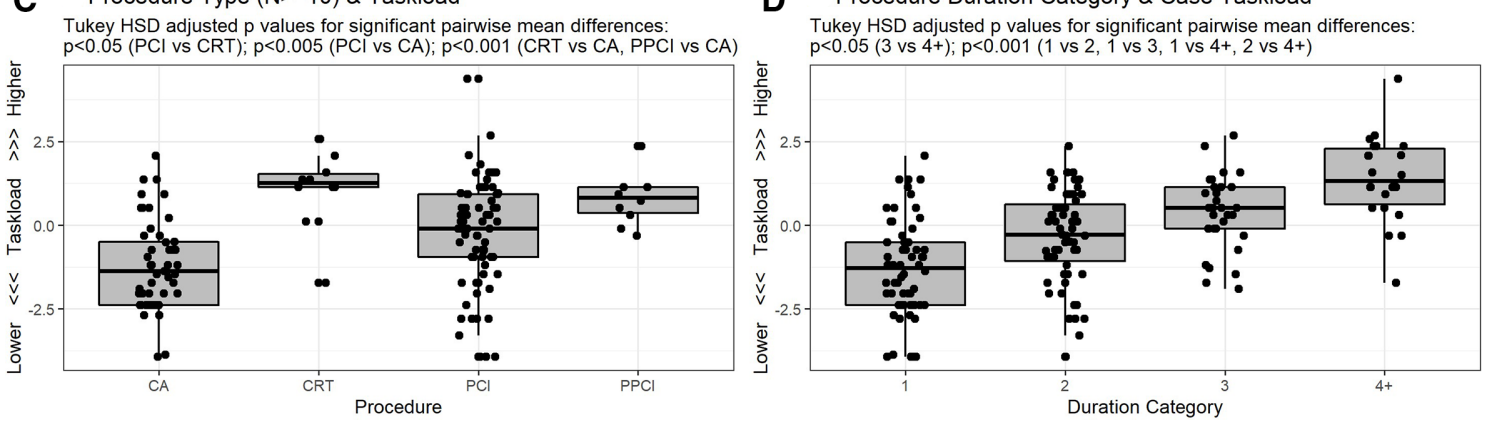

Figure 2 The relationship between task load and case variables. (A) task load in the presence and absence of distractions; (B) number of distractions per procedure and task load; (C) procedure types and task load; (D) procedure duration and task load. CA, coronary angiography; CRT, cardiac resynchronisation therapy; HSD, Honest Significant Difference; PCI, percutaneous coronary intervention; $\mathrm{PPCl}$, primary percutaneous coronary intervention.

'distraction' group was 9.5 vs 9.7 in the 'no distraction' group. Patient perception of team continuity throughout procedure was recorded in $92 \%(\mathrm{n}=97)$ in the 'distraction' group and $97 \%(\mathrm{n}=85)$ in the 'no distraction' group. Catheter laboratory team continuity was perceived by $89 \%$ of patients in the 'distraction' group and $98 \%$ in the 'no distraction' group.

\section{DISCUSSION}

Our results demonstrate the magnitude and frequency of distractions and interruptions during a range of invasive cardiac procedures, in a high volume district general hospital, with fewer than half of all procedures being completed without interruption/distraction. The majority of interruptions are irrelevant and non-urgent, causing unnecessary disruption to catheter laboratory team members, operators and in some cases resulting in transient but complete cessation of procedures. This raises patient safety concerns.

These findings share similarity with surgical data. For example, 85 distracting communications and 52 other types of distractions were seen during 24 urological procedures. ${ }^{8}$ In a study of 65 surgical procedures (excluding those with duration $>4$ hours) 803 interruptions/distractions occurred (averaging 12 interruptions/procedure) ${ }^{13}$ The higher frequency of distractions observed in these studies, compared with ours, might in part be explained by the use of dedicated trained observers.

Data from cognitive psychology research demonstrate that on interruption, the focus of an individual shifts from the primary task (in this case cardiac procedure) to the subject of the distraction. On resumption of the primary task a cognitive memory lag ensues, whereby the individual may transiently forget what stage of the primary procedure they had just completed or their planned strategy to successfully complete a procedural step. ${ }^{14}$ Clinician multitasking has negative effects on clinical task completion: 40 emergency departments doctors were observed to have $11 \%$ of their tasks interrupted and failure to return to the original task was seen in $18.5 \%$ of these cases. ${ }^{15}$ In an experimental setting of radiologists interpreting CT chest images, interruptions led to increase in task completion time by $8-13 \%$ but without increase in errors. ${ }^{16}$ A study of ear, nose and throat surgeons performing a simulated surgical task demonstrated that interruptions precipitated both an increased time to task completion and frequency of errors. ${ }^{17}$ In the context of a complex or critical stage of a cardiac procedure cognitive disruption due to task switching or multi-tasking may have significant implications both to successful procedural completion and patient safety. Furthermore it has been suggested that attendance to a new task may increase the risk of error within one or both tasks due to induced cognitive fatigue potentially leading to multiple suboptimal clinical decisions. ${ }^{18}$

Our data demonstrate an association between increased operator task load and procedural distractions. Fifty eight per cent of procedures were performed by senior operators (consultants) and previously reported data suggest that experienced operators are less likely to be negatively affected by distractions. ${ }^{17} 19$ Interestingly, within the Rasch model, as overall task load increased the 
component attracting higher ratings was mental workload. It is tempting to speculate that operator distraction impacts on cognition above and beyond other task load components. This finding differs from a study of associations between interruptions and surgeons' perceived workload during 56 general surgical/orthopaedic cases. ${ }^{20}$ Case-irrelevant communications were associated with increased intraoperative distraction but less with mental fatigue/stress.

Effort was the second component to rise, supporting the concept that increasing demand on cognition is associated with operator perception of increased effort. Operator frustration and performance were the last components to rise with task load. We speculate that this may be explained by operator conditioning. Cardiologists are trained to remain calm under pressure and hence although subject to increased mental workload they may be able to control frustration to maintain an adequate level of performance. However, through learning lessons from aviation and surgical colleagues perhaps it is time to rely less on the cognitive multitasking capabilities of clinicians and rather focus on the facilitation of a safer and more conducive catheter laboratory environment.

Unsurprisingly the longer the procedure the more likely it will be interrupted. In our study interruptions were observed in all 20 procedures lasting $>120 \mathrm{~min}$. Longer procedures were also associated with increased task load. Apart from minimising distractions in general it seems sensible for both operator and team members to schedule breaks at non critical times of longer procedures. It is interesting (noting that sedation is often given for procedures) that distractions did not impact on patients' perception of procedural success.

Could the introduction of a 'sterile cockpit concept' within the cardiac catheter laboratories improve both working conditions and patient safety? First described in aviation, the 'sterile cockpit' mandates only essential flight-relevant communication and no interruptions at any altitude below 10000 feet. This ensures crew members are focused on their tasks during the most critical stages of flight. Wadhera et al demonstrated that use of communication analogous to the 'sterile cockpit' led to a positive effect on overall communication with a reduction in communication failures during cardiac surgery. ${ }^{21}$ We advocate the introduction of the 'sterile cockpit concept' into the WHO team brief at the start of every procedural list, whereby, at any critical procedural phase, any team member (though most likely the primary operator) can instigate a 'sterile cockpit' environment. This would automatically trigger all cardiac catheter laboratory team members to revert to case relevant essential communication, to become hyper-alert to both patient and environmental factors that may impact on safety or procedural outcome and to minimise distractions both within and external to the catheter laboratory. Invoking the 'sterile cockpit' is not analogous to 'muting' catheter laboratory staff-rather the contrary. Extensive research has shown the dangers of steep hierarchical boundaries both in aviation and within healthcare. ${ }^{22}$ Hence, the 'sterile cockpit' is not a tool by which the operator demands silence but rather an instrument to effectively focus the team, ensuring heightened awareness to a clinically deteriorating patient, challenging intervention or potential complication, and in tandem, empowering the team to communicate relevant observations or concerns that may warrant imminent action.

\section{Limitations}

Limitations of our study include that it is a single centre experience over a relatively short time period. Though it reflects real-world practice, real-time data collection was performed by cardiac physiologists in addition to their clinical duties, as we did not have a dedicated observer. Although key data was comprehensively collected, additional information such as risk stage at the time of distraction was only captured in a third of cases. Mindful that the exercise of data collection should not itself become a distraction to the physiologist from their primary role, we exercised significant flexibility around data collection particularly for free-text fields. As such it is likely that we have underestimated the interruption burden. We cannot account for the Hawthorne effect (when being observed leads to change in behaviour) - it is feasible that through the study period, individuals (both within and external to the catheter laboratory) may have adopted behavioural changes to reduce unnecessary distractions. Despite this our data clearly demonstrates high volume non-urgent and irrelevant interruptions throughout the study period.

\section{CONCLUSION}

To our knowledge, this is the first description of HF in the adult cardiac catheterisation laboratory. We have shown that fewer than half of all procedures are completed without interruption/distraction. The vast majority of these are unnecessary and are associated with increased operator task load. We, therefore, propose the introduction of a 'sterile cockpit' environment in the catheter laboratory, as has been adapted from aviation and is used within surgical operating theatres, to minimise nonemergent interruptions and disturbances, in an attempt to improve both catheterisation laboratory working conditions and overall patient safety.

Twitter Kalaivani Mahadevan @KVM83, Elena Cowan @ElenaCowan_and Peter A Brennan @BrennanSurgeon

Contributors KM: data acquisition, analysis and interpretation, manuscript drafting and critical revision. EC: data analysis and interpretation, critical revision of manuscript. NK: study design. HB: data acquisition. RA: data analysis and interpretation, critical revision of manuscript. AH: data acquisition and interpretation, critical revision of manuscript. KG: data acquisition and interpretation, critical revision of manuscript. GM: data acquisition and interpretation, critical revision of manuscript. PAB: data interpretation, critical revision of manuscript. PRK: study conception and design, data acquisition and interpretation, critical revision of manuscript. All authors have contributed to the revision of manuscript and have accepted the final version. Guarantors: KM, PRK.

Funding The authors have not declared a specific grant for this research from any funding agency in the public, commercial or not-for-profit sectors. 
Competing interests None declared.

Patient consent for publication Not required.

Ethics approval This was a combined patient safety and service evaluation project without impact on standard patient care in accordance with the UK Policy Framework for Health and Social Care Research guidance, hence formal Research Ethic Committee approval and consent were not required.

Provenance and peer review Not commissioned; externally peer reviewed.

Data availability statement All data relevant to the study are included in the article or uploaded as online supplemental information.

Open access This is an open access article distributed in accordance with the Creative Commons Attribution Non Commercial (CC BY-NC 4.0) license, which permits others to distribute, remix, adapt, build upon this work non-commercially, and license their derivative works on different terms, provided the original work is properly cited, appropriate credit is given, any changes made indicated, and the use is non-commercial. See: http://creativecommons.org/licenses/by-nc/4.0/.

ORCID iDs

Kalaivani Mahadevan http://orcid.org/0000-0002-9414-6945

Elena Cowan http://orcid.org/0000-0003-0491-374X

\section{REFERENCES}

1 Powell-Dunford N, Brennan PA, Peerally MF, et al. Mindfu application of aviation practices in healthcare. Aerosp Med Hum Perform 2017;88:1107-16.

2 Green B, Tsiroyannis C, Brennan PA. Human factors--recognising and minimising errors in our day to day practice. Oral Dis 2016;22:19-22.

3 Kellogg KM, Puthumana JS, Fong A, et al. Understanding the types and effects of clinical interruptions and Distractions recorded in a multihospital patient safety reporting system. J Patient Saf 2018. doi:10.1097/PTS.0000000000000513

4 Willett M, Gillman O, Shin E, et al. The impact of distractions and interruptions during cesarean sections: a prospective study in a London teaching hospital. Arch Gynecol Obstet 2018;298:313-8.

5 Gillespie BM, Harbeck E, Kang E, et al. Correlates of non-technical skills in surgery: a prospective study. BMJ Open 2017;7:e014480.

6 Westbrook JI, Raban MZ, Walter SR, et al. Task errors by emergency physicians are associated with interruptions, multitasking, fatigue and working memory capacity: a prospective, direct observation study. BMJ Qual Saf 2018;27:655-63.

7 Mentis HM, Chellali A, Manser K, et al. A systematic review of the effect of distraction on surgeon performance: directions for operating room policy and surgical training. Surg Endosc 2016;30:1713-24.
8 Sevdalis N, Undre S, McDermott $\mathrm{J}$, et al. Impact of intraoperative distractions on patient safety: a prospective descriptive study using validated instruments. World J Surg 2014;38:751-8.

9 Westbrook JI, Li L, Hooper TD, et al. Effectiveness of a 'Do not interrupt' bundled intervention to reduce interruptions during medication administration: a cluster randomised controlled feasibility study. BMJ Qual Saf 2017;26:734-42.

10 Cahill TJ, Clarke SC, Simpson IA, et al. A patient safety checklist for the cardiac catheterisation laboratory. Heart 2015;101:91-3.

11 Healey AN, Primus CP, Koutantji M. Quantifying distraction and interruption in urological surgery. Qual Saf Health Care 2007;16:135-9.

12 Bergersen L, Gauvreau K, Marshall A, et al. Procedure-type risk categories for pediatric and congenital cardiac catheterization. Circ Cardiovasc Interv 2011;4:188-94.

13 Antoniadis S, Passauer-Baierl S, Baschnegger $\mathrm{H}$, et al. Identification and interference of intraoperative distractions and interruptions in operating rooms. J Surg Res 2014;188:21-9.

14 Altman EM, Trafton JG. Task interruption: resumption lag and the role of cues. Proceedings of the 26th Annual Conference of the Cognitive Science Society, 2004:42-7.

15 Westbrook JI, Coiera E, Dunsmuir WTM, et al. The impact of interruptions on clinical task completion. Qual Saf Health Care 2010;19:284e289.

16 Williams LH, Drew T. Distraction in diagnostic radiology: how is search through volumetric medical images affected by interruptions? Cogn Res Princ Implic 2017;2:12.

17 Ahmed A, Ahmad M, Stewart CM, et al. Effect of distractions on operative performance and ability to multitask - a case for deliberate practice. Laryngoscope 2015;125:837-41. doi:10.1002/ lary.24856

18 Grissinger M. Sidetracks on the safety express: interruptions lead to errors and ... wait, what was I doing? P T 2015;40:145-90.

19 Park J, Waqar S, Kersey T, et al. Effect of distraction on simulated anterior segment surgical performance. $J$ Cataract Refract Surg 2011;37:1517-22.

20 Weigl M, Antoniadis S, Chiapponi C, et al. The impact of intraoperative interruptions on surgeons' perceived workload: an observational study in elective general and orthopedic surgery. Surg Endosc 2015;29:145-53

21 Wadhera RK, Parker SH, Burkhart HM, et al. Is the "sterile cockpit" concept applicable to cardiovascular surgery critical intervals or critical events? The impact of protocol-driven communication during cardiopulmonary bypass. J Thorac Cardiovasc Surg 2010;139:312-9.

22 Brennan PA, Davidson M. Improving patient safety: we need to reduce hierarchy and empower junior doctors to speak up. BMJ 2019;366:I4461. 